Vestibuläres Rehabilitationstraining

\title{
Physiotherapie stoppt den Schwindel
}

Patienten mit chronischem Schwindel, ausgelöst durch eine Schädigung des Gleichgewichtsorgans oder des achten Hirnnerven, z. B. nach Neuritis vestibularis oder eines Morbus Menière, profitieren deutlich von vestibulärem Rehabilitationstraining.

Den Mehraufwand der Therapie rechtfertigt nicht nur die klinische Verbesserung der Schwindelsymptomatik, sondern auch die Kosteneffektivtät für das Gesundheitssystem. Dies zeigte eine Studie mit 337 Patienten aus 35 Arztpraxen.

Untersucht wurde die Wirksamkeit und der ökonomischen Nutzen eines physiotherapeutischen Trainingsprogramms. Die Patienten erhielten entweder die Standardtherapie ohne Physiotherapie oder ein vestibuläres Rehabilitationstraining anhand einer Trainingbroschüre mit oder ohne der Möglichkeit einer Unterstützung am Telefon. Primärer Endpunkt war die Vertigo Symptoms Scale (VSS) nach zwölf Wochen und nach einem Jahr sowie Schwindel pro qualitätskorrigiertem Lebensjahr. Es zeigte sich eine deutliche Überlegenheit des vestibulären Rehabilitationstrainings im Vergleich zur Standardtherapie, wobei die Patienten mit der Möglichkeit einer zusätzlichen Telefonunterstützung insgesamt am meisten profitierten. Auch zeigte sich, dass beide Interventionen kosteneffektiv waren, wobei es hierbei offenbar einen positiven Einfluss der zusätzlichen Telefonhilfe gab, da diese Patienten am kosteneffektivsten von allen drei Gruppen waren.

Yardley L et al, Clinical and cost effectiveness of booklet based vestibular rehabilitation for chronic dizziness in primary care. BMJ 2012, 344: e2237
KOMMENTAR

PD Dr. Mark Obermann

Wissenschaftlicher und klinischer Leiter des Schwindel-Zentrums Essen

\section{Unterstützung zahlt sich aus}

Der Zugang für Patienten mit chronischem Schwindel zu spezialisierten Schwindel-Zentren, die vestibuläres Rehabilitationstraining mit ausgebildeten Physiotherapeuten anbieten, ist nicht nur in England sehr limitiert.

Wie diese Studie eindrucksvoll zeigt, reicht ein wenig Unterstützung durch Ausgabe spezieller Trainingsbroschüren mit adäquater Anleitung der einzelnen Übungen aus, um eine deutliche Verbesserung der chronischen Schwindelsymptomatik dieser meist langjährig erkrankten und stark eingeschränkten Patienten zu bewirken.

Neben der Verbesserung der Lebensqualität des einzelnen Patienten scheint es darüber hinaus auch noch wesentlich günstiger für unser $\mathrm{Ge}$ sundheitssystem zu sein.

\section{Akuter idiopathischer Hörsturz}

\section{Kortikosteroide sind unwirksam}

Dass Kortikosteroide in der Therapie des akuten idiopathischen Hörsturzes unwirksam sind, zeigte eine schwedische Studie.
Von den 93 Patienten im Alter von 18-80 Jahren, die einen plötzlichen Hörsturz - definiert als eine Hörminderung um mehr als $30 \mathrm{~dB}$ - hatten, erhielten 47 Patienten Pred-

\section{KOMMENTAR}

Prof. Dr. Hans-Christoph Diener

Direktor der Klinik für Neurologie, Universitätsklinikum Essen

\section{Bemerkenswertes Ergebnis}

Diese wichtige und gut durchgeführte Studie zeigt, dass Kortikosteroide zur Behandlung des idiopathischen Hörsturzes unwirksam sind. Dies ist bemerkenswert, da sowohl für die Neuritis vestibularis als auch für die periphere Facialisparese eine
Wirkung von Kortikosteroiden gegenüber Placebo nachgewiesen wurde. $\mathrm{Ob}$ dies nun durch eine unterschiedliche $\mathrm{Pa}$ thophysiologie oder durch die relativ geringe Zahl der Patienten in der Studie bedingt ist, bleibt ungeklärt. nisolon und 46 Placebo. Etwa zwei Drittel der Patienten klagten initial über Tinnitus, ein Viertel über Drehschwindel. Die Therapie begann mit $60 \mathrm{mg}$ Prednisolon für drei Tage, dann wurde täglich um $10 \mathrm{mg}$ reduziert. Patienten, die nach Tag 8 noch Hörstörungen hatten, wurden bis Tag 30 mit $10 \mathrm{mg}$ Prednisolon täglich weiter behandelt. Primärer Endpunkt war die Verbesserung des Hörvermögens am Tag 90 . Nach acht Tagen hatte sich das Hörvermögen in der Prednisolon-Gruppe um $25 \mathrm{~dB}$ verbessert, in der Placebogruppe um $26 \mathrm{~dB}$. Nach 90 Tagen um $39 \mathrm{~dB}$ und um $35 \mathrm{~dB}$ mit Placebo. Beide Unterschiede waren statistisch nicht signifikant. Die Studie zeigte auch, dass begleitender Drehschwindel und Entzündungszeichen im Labor negative Prädiktoren für ein gutes Outcome waren.

Nosrati-Zarenoe R, Hultcrantz E, Otol Neurotol 2012, 33: 523 